\title{
Notch in T-ALL: new players in a complex disease
}

\section{Ute Koch and Freddy Radtke}

Ecole Polytechnique Fédérale de Lausanne (EPFL), Swiss Institute for Experimental Cancer Research (ISREC), Station 19, 1015 Lausanne, Switzerland

T cell acute lymphoblastic leukemia (T-ALL) is an aggressive hematopoietic malignancy of thymocytes affecting preferentially children and adolescents. The disease is heterogeneous and characterized by a large set of chromosomal and genetic alterations that deregulate the growth of maturing thymocytes. The identification of activating point mutations in NOTCH1 in more then $50 \%$ of all T-ALL cases highlights the NOTCH1 cascade as a central player of T-ALL pathogenesis. In this review, we summarize and update more recent findings on the molecular mechanisms of T-ALL with a particular emphasis on the oncogenic properties of aberrant NOTCH1 signaling.

\section{Notch signaling finds its place in T-ALL}

$\mathrm{T}$ cell acute lymphoblastic leukemia (T-ALL) is an aggressive malignancy of developing thymocytes. The disease represents $15 \%$ of pediatric and $25 \%$ of adult acute lymphoblastic leukemia (ALL) cases. It is characterized clinically by high white blood cell counts, increased numbers of blast cells and enlarged mediastinal lymph nodes. Although the disease develops mostly in the thymus, it tends to spread throughout the body, including the central nervous system, complicating the therapeutic treatment of the disease. Historically, T-ALL was associated with a poor outcome; however, today's more aggressive multi-agent chemotherapy results in an overall survival rate of $70 \%$ for children and 30-40\% for adults below 60 years of age, and $10 \%$ above this age [1]. Relapse patients often develop resistance to chemotherapy, and are associated with very poor prognosis. It is therefore important to understand the molecular mechanisms that cause and drive T-ALL to identify novel molecular targets and design more specific therapies.

Over the last two decades, cytogenetic analysis, combined with cloning and sequencing of chromosomal translocation break points, revealed that aberrant expression of numerous transcriptional regulators in T-ALL often involves incorrect rearrangement processes of the $\mathrm{T}$ cell receptor (TCR) genes. This results in the juxtaposition of genes coding for transcriptional regulators close to strong TCR gene enhancers and promoters that drive their expression [2]. Examples include a number of basic helixloop-helix (bHLH) transcription factors such as TAL1, TAL2, LYL1, bHLHB1 and MYC, or homeobox transcription factors (Table 1). Although some of the transcriptional

Corresponding author: Radtke, F. (Freddy.Radtke@epfl.ch) regulators (e.g. MYC) are expressed in normal thymocytes where they govern $\mathrm{T}$ cell differentiation and development, others are not of lymphoid origin (e.g. TAL1 or TAL2).

Additional chromosomal translocations, which do not implicate the TCR rearrangement machinery with their strong promoters and enhancers, have also been identified in T-ALL patients. These non-TCR associated translocations lead to the generation of fusion genes and chimeric proteins with novel functions, gene deletions, or gene inactivation as a consequence of promoter hypermethylation, as well as gene duplications (Table 2).

Although it is in many instances unclear how all of these different genetic defects mechanistically transform thymocytes, this incomplete list shows the large variety of genetic aberrations that contribute to the transformation of T cells. T-ALL does not represent a single disease entity. Gene expression profiling, as well as microarray based competitive genomic hybridization (array-CGH), which detects genomic copy number variations such as deletions and/ or duplications, helped to classify T-ALL patients into multiple subgroups. Some of these subgroups exhibit unique aberrations, whereas others are shared by multiple patient subgroups [3,4]. More than $50 \%$ of T-ALL patients have mutations leading to the hyperactivation of the Notch1 pathway, suggesting that this signaling cascade plays an important role in T-ALL pathogenesis. Box 1

Notch1 is one of four single transmembrane bound Notch receptors, which are composed of an extracellular and an intracellular subunit that are non-covalently linked through the heterodimerization domain (HD) (Figure 1). Notch signaling is initiated by receptor-ligand interaction between two neighboring cells, leading to successive proteolytic cleavages, which result in the liberation of the intracellular domain of the receptors (NICD). NICD then translocate to the nucleus and heterodimerizes with the transcription factor CSL/RBPJK to regulate gene expression [5]. This signaling cascade is evolutionarily conserved and regulates many cellular processes, including proliferation, differentiation and survival. Here we summarize recent findings on the differences between human and murine oncogenic Notch1 mutations in T-ALL, and discuss Notch targets and downstream signaling pathways.

\section{Notch1 - key player in T-ALL}

Notch signaling through the Notch1 receptor has long been shown to be essential for $\mathrm{T}$ cell lineage development, thymocyte survival, as well as the proliferation of committed $\mathrm{T}$ cell progenitors. Historically, the first evidence of 
Table 1. TCR-mediated translocations in T-ALL.

\begin{tabular}{|c|c|c|c|c|c|}
\hline Genes & Function & Rearrangements & Frequency & Outcome & References \\
\hline TAL2 & $\begin{array}{l}\text { bHLH transcription factor } \\
\text { Neural development }\end{array}$ & $\begin{array}{l}\text { TCR } \beta \\
t(7 ; 9)(q 35 ; q 34)\end{array}$ & $<2 \%$ & Unknown & {$[50,51]$} \\
\hline LYL1 & $\begin{array}{l}\text { bHLH transcription factor } \\
\text { HSCs, B cell development, vasculogenesis }\end{array}$ & $\begin{array}{l}\text { TCR } \beta \\
t(7 ; 19)(q 35 ; p 13)\end{array}$ & $<2 \%$ & Unknown & [51] \\
\hline HBHL1/OLIG2 & $\begin{array}{l}\text { bHLH transcription factor } \\
\text { Neural development } \\
\text { Oligodendrocyte differentiation }\end{array}$ & $\begin{array}{l}\text { TCR } \alpha \\
t(14 ; 21)(q 11 ; q 22)\end{array}$ & Rare & Unknown & [52] \\
\hline C-MYC & $\begin{array}{l}\text { bHLH/ZIP transcription factor, cell growth, } \\
\text { apoptosis, stem cell self-renewal and } \\
\text { differentiation }\end{array}$ & $\begin{array}{l}\text { TCR } \alpha \\
\mathrm{t}(8 ; 14)(\mathrm{q} 24 ; \mathrm{q} 11)\end{array}$ & $2 \%$ & Poor & [53-55] \\
\hline LMO1 & $\begin{array}{l}\text { LIM-domain containing transcriptional regulators } \\
\text { Megakaryocyte/Erythrocyte differentiation }\end{array}$ & $\begin{array}{l}\text { TCR } \alpha / \delta \\
t(11 ; 14)(p 15 ; q 11)\end{array}$ & $<1 \%$ & Unknown & {$[56,57]$} \\
\hline LMO2 & $\begin{array}{l}\text { LIM-domain containing transcriptional regulators } \\
\text { Megakaryocyte/Erythrocyte differentiation }\end{array}$ & $\begin{array}{l}\text { TCR } \alpha / \delta \\
\text { t(11;14)(p13;q11) } \\
\text { TCR } \beta \\
t(7 ; 11)(q 35 ; p 13)\end{array}$ & $\begin{array}{l}7 \% \\
3 \%\end{array}$ & $\begin{array}{l}\text { Unknown } \\
\text { Unknown }\end{array}$ & {$[58,59]$} \\
\hline Hox11/TLX1 & $\begin{array}{l}\text { Homeodomain transcription factor } \\
\text { Spleen development }\end{array}$ & $\begin{array}{l}\text { TCR } \alpha / \delta \\
\mathrm{t}(10 ; 14)(q 24 ; q 11) \\
\text { TCR } \beta \\
\text { t(7;10)(q34;q24) }\end{array}$ & $5-10 \%$ & Good & [60-62] \\
\hline Hox11L2/TLX3 & $\begin{array}{l}\text { Homeodomain transcription factor } \\
\text { Neural development }\end{array}$ & $\begin{array}{l}\text { TCR } \alpha / \delta \\
(\mathrm{t}(5 ; 7)(\mathrm{q} 35 ; \mathrm{q} 21)\end{array}$ & $24 \%$ & $\begin{array}{l}\text { Poor } \\
\text { No impact }\end{array}$ & {$[60,63]$} \\
\hline C-MYB & Transcription factor, hematopoiesis & $\begin{array}{l}\text { TCR } \beta \\
t(6 ; 7)(q 23 ; q 34)\end{array}$ & $3 \%$ & Unknown & [64] \\
\hline $\mathrm{NOTCH} 1$ & $\begin{array}{l}\text { Type I receptor, T cell commitment and } \\
\text { thymocyte maturation }\end{array}$ & $\begin{array}{l}\text { TCR } \beta \\
t(7 ; 9)(q 34 ; q 34.3)\end{array}$ & $<1 \%$ & Good & [6] \\
\hline
\end{tabular}

Table 2. Non-TCR-mediated translocations and mutations in T-ALL.

\begin{tabular}{|c|c|c|c|c|c|}
\hline Genes & Function & Rearrangements & Frequency & Outcome & References \\
\hline STIL/TAL1 & $\begin{array}{l}\text { bHLH transcription factor } \\
\text { HSC survival, Megakaryocyte/Erythrocyte differentiation }\end{array}$ & 1p32 deletion & $4 \%$ & Good & [50] \\
\hline CDKN2A/2B & Cell cycle regulator & $\begin{array}{l}9 \mathrm{p} 21 \text { deletions or } \\
\text { hypermethylation }\end{array}$ & $70 \%$ & Poor & {$[65,66]$} \\
\hline Hox A cluster & Regulation of axial patterning during development & $\operatorname{inv}(7)(p 15 q 34)$ & $3 \%$ & Unknown & {$[67,3]$} \\
\hline Hox11L2 & $\begin{array}{l}\text { Homeodomain transcription factor } \\
\text { Neural development }\end{array}$ & $\begin{array}{l}\text { BCL11B } \\
t(5 ; 14)(q 35 ; q 32) \\
\text { CDK6 } \\
(t(5 ; 7)(q 35 ; q 21)\end{array}$ & $24 \%$ & No impact & {$[60,63]$} \\
\hline C-MYB & Transcription factor, hematopoiesis & Gene duplication & $8-15 \%$ & Unknown & {$[64,68]$} \\
\hline EML1-ABL1 & $\begin{array}{l}\text { EML1-Cytoskeleton } \\
\text { ABL-Nuclear tyrosine kinase, regulating cell } \\
\text { differentiation and division }\end{array}$ & Gene fusion & $<1 \%$ & Unknown & [69] \\
\hline ETV6-ABL1 & $\begin{array}{l}\text { ETV6-Ets-family transcription factor, hematopoiesis } \\
\text { ABL-Nuclear tyrosine kinase, regulating cell } \\
\text { differentiation and division }\end{array}$ & Gene fusion & $<1 \%$ & Unknown & [70] \\
\hline NOTCH1 & $\begin{array}{l}\text { Type I receptor, T cell commitment } \\
\text { and thymocyte maturation }\end{array}$ & Mutations & $>50 \%$ & Good & [11] \\
\hline FBXW7 & Ubiquitin ligase, regulating protein degradation & Mutations or deletions & $8-15 \%$ & Poor & {$[74,75]$} \\
\hline PTEN & Phosphatase, negative regulator of PI3K-Akt signaling & $\begin{array}{l}\text { Mutations } \\
\text { Deletions }\end{array}$ & $\begin{array}{l}27 \% \\
9 \%\end{array}$ & Poor or no impact & {$[76,77,22]$} \\
\hline
\end{tabular}




\section{Box 1. Therapeutic targeting of the Notch pathway}

The best-documented causative role of activated Notch signaling in human carcinogenesis is certainly $\mathrm{T}$ cell acute lymphoblastic leukemia (T-ALL). In recent years aberrant Notch signaling has also been linked to various forms of solid human tumors, and thus, has gained increasing attention as a potential therapeutic target. Initial strategies to block Notch signaling that were also explored in clinical trials, were to use small molecule inhibitors of the $\gamma$-secretase complex [78]. These chemical compounds block the proteolytic cleavage that liberates the intracellular Notch domain (NICD). However, $\gamma$-secretase inhibitors are not selective for individual Notch receptors, but they block signaling of all receptors. The most frequently observed side-effect of these compounds is that they cause a dose dependent goblet cell metaplasia in the intestine [79], since inhibition of Notch1 and Notch2 in intestinal crypt progenitors and/or stem cells induces premature differentiation into goblet cells [80]. However, the gut toxicity can be circumvented when these $\gamma$ secretase inhibitors are used in combinatorial therapy with glucocorticoids such as dexamethasone [81]. Alternative strategies employing blocking antibodies for specific Notch receptors and/or ligands are being developed currently [81-85]. The advantage of inhibiting selectively individual Notch receptors or ligands is the minimization of potential side effects. Preclinical cell culture and xeno-transplantation studies indeed indicate that antibody-mediated inhibition of Notch signaling is a promising avenue [83]. Nevertheless, this approach has its limitations. The activity of certain antibodies to block the growth of human T-ALL cell lines bearing Notch 1 mutations appears to be less efficient compared to $\gamma$-secretase inhibitors [86], possibly owing to lower binding affinity to the mutated receptor. Moreover, tumor cells harboring chromosomal translocations or genetic aberrations, which lead to the expression of Notch receptors lacking their extracellular domain, are not treatable with an antibody-mediated approach. Therefore, alternative approaches must be considered, and were recently pioneered successfully. Stabilized small $\alpha$-helical peptides derived from the mastermind-like protein were employed to inhibit the Notch transcription complex. These peptides bind to the groove formed by the CSL-NICD complex, and thereby prevent the recruitment of necessary transcriptional coactivators [87]. Treatment of leukemic cells with such peptides inhibited the proliferation of leukemic cells in vitro as well as in a Notch1-driven T-ALL mouse model without causing gut toxicity. Whether any of these or other strategies will be developed successfully into a clinical drug, or will be used in combined drug therapies, remains to be assessed in the future.

Notch1 being involved in T-ALL was provided when NOTCH1 was identified through the analysis of a chromosomal translocation [t(7;9)(q34; 34.3$)]$ detected in a small number of T-ALL patients. This revealed that a portion of the human NOTCH1 gene had translocated into the TCRB locus [6,7]. The oncogenic potential of NOTCH1 was demonstrated in murine bone marrow (BM) reconstitution experiments. Mice reconstituted with BM cells expressing a truncated human form of NOTCH1 developed hematological malignancies characterized as T-ALL $[8,9]$. However, the role of NOTCH1 in human T-ALL seemed to be limited because of the rare number of patient cases identified with the $t(7 ; 9)$ translocation (less than $1 \%$; [10]). A landmark study published in 2004 changed the situation dramatically by showing that more than $50 \%$ of T-ALL cases carry Notch1-activating mutations [11]. This finding implicated that Notch1 has a very prominent role in the pathogenesis of T-ALL. Sequence analysis of primary human T-ALL samples and human T-ALL cell lines revealed that the majority of these mutations cluster in 2 general regions either at the PEST or the HD domain of Notch1. The mutations clustering at the C-terminus of the Notch1

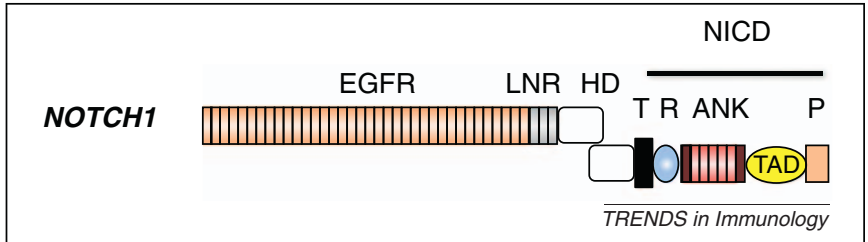

Figure 1. Schematic representation of the human Notch1 receptor. The extracellular domain of Notch1 consists of 36 epidermal growth factor (EGF)-like repeats (EGFR) followed by three cysteine-rich lin12/Notch repeats (LNR) and the heterodimerization domain (HD). Notch1 contains a single transmembrane domain (T) followed by a RBPJk-associated module (R) domain and 6 ankyrin repeats (ANK) flanked by two nuclear localization signals, a transactivating domain (TAD), and a PEST sequence $(\mathrm{P})$ involved in regulating protein turnover.

receptor consist of nonsense or frameshift mutations resulting in the deletion of the PEST domain normally regulating NICD degradation [12]. These mutations increase Notch activity through the stabilization of NICD and are present in approximately $20-30 \%$ of tumors. The most common NOTCH1 mutations $(40-45 \%$ of T-ALL patients) fall into exon 26 or 27 , which encode the $\mathrm{N}$ and C-terminal HD subunits. Mutations within that region consist of single amino acid changes, short insertions or deletions that maintain the reading frame. These mutations destabilize the interaction between the two subunits, and induce or facilitate ligand-independent Notch1 signaling [13]. Most Notch-dependent human cell lines and approximately $10-20 \%$ of primary human T-ALL reveal mutations in both the HD and PEST domains (Figure 2a).

In retrospect it is not surprising that the frequently occurring mutations in the human NOTCH1 receptor have been associated with human T-ALL given that Notch has been shown to be a potent oncogene in mouse models. Retroviral or transgenic overexpression of NICD in hematopoietic or $\mathrm{T}$ cell progenitors is widely used to induce T-ALL in mice $[9,14,15]$. However, sporadic mutations in murine and human T-ALL have only been found so far in Notch1 suggesting that Notch1, and not other Notch receptors, can be a major driver in human T-ALL. Although many T-ALL tumors harbor mutations within the NOTCH1 gene, they contain in addition chromosomal translocations or rearrangements that activate oncogenes or create oncogenic fusion genes (see Tables 1 and 2). Thus, the question remained whether aberrant Notch signaling is the driver or only a cooperating passenger in human T-ALL.

Although NICD1 is a potent inducer of T-ALL in retroviral and transgenic mouse models, this form of Notch is rarely present in human T-ALL. In mouse models of TALL, such as TAL1/SCL, OLIG2 and LMO1/2 transgenic mice (see Table 1 and [16] for review), spontaneous activating Notch1 mutations occur at a high frequency. Therefore, the more commonly mutated Notch 1 alleles in the retroviral mouse model have been analyzed. HD, PEST, and HD/PEST mutations more frequently found in human T-ALL samples were weak inducers of Notch activity in vitro, induced only ectopic $\mathrm{T}$ cell development, and failed to induce leukemia when tested in the retroviral mouse model [17]. Once these constructs were assayed in an oncogenic background that predisposes to T-ALL, using the LSL-K-ras ${ }^{\mathrm{G} 12 \mathrm{D}}$ mouse strain, these weakly leukemogenic alleles shortened disease latency and gave rise to cell 


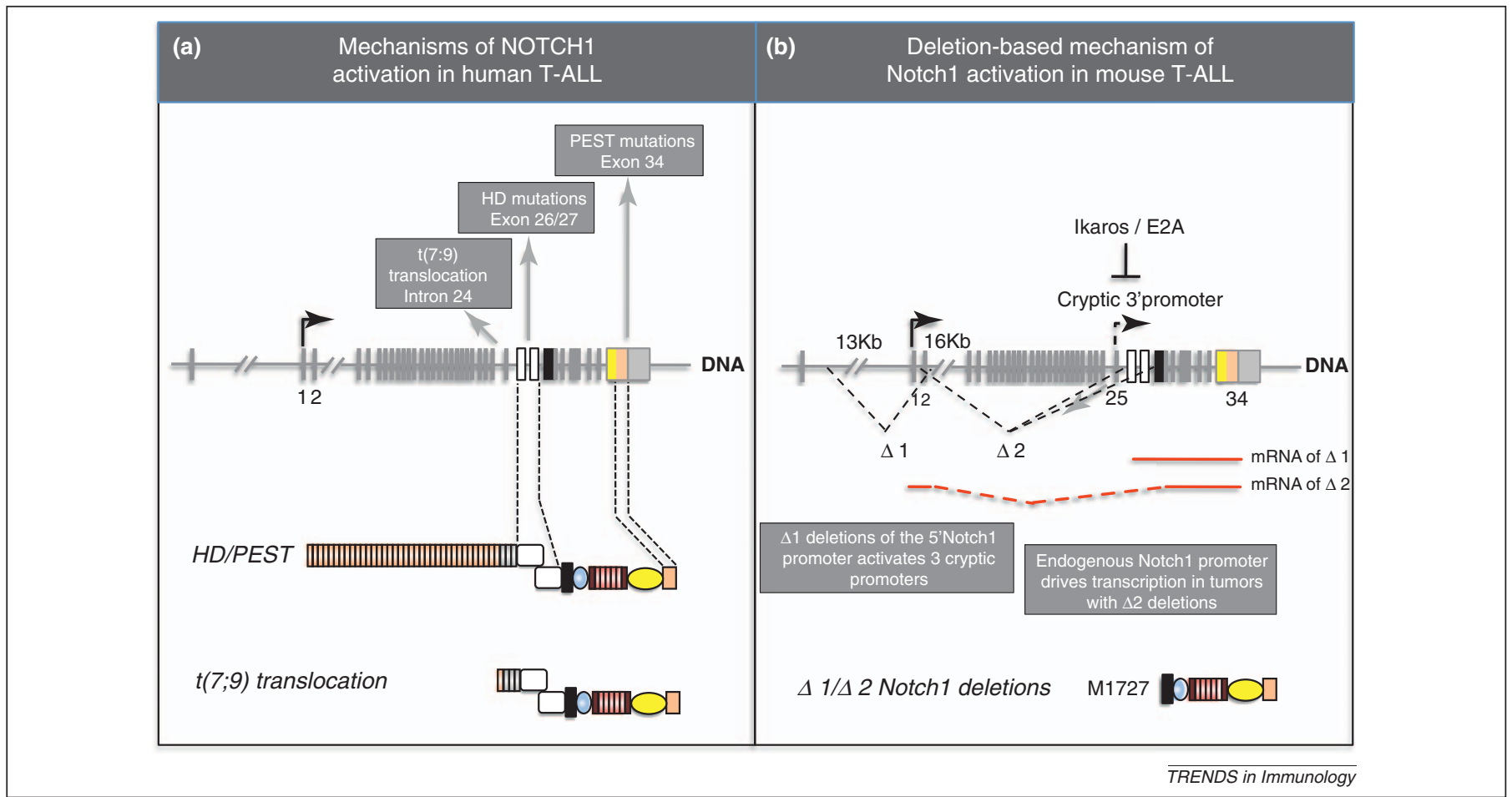

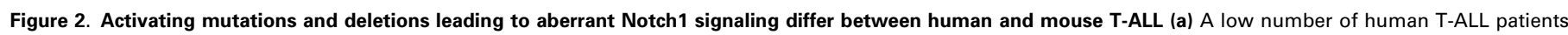
carry a $t(7 ; 9)$ translocation, which was mapped to intron 24 and results in the generation of a truncated dominant active Notch1 receptor lacking most of its extracellular domain. Most of the NOTCH1 mutations in humans localize either to the HD domain or to the PEST domain, whereas some T-ALL patients reveal mutations in both domains. In the schematic of NOTCH1, bars and numbers indicate exons and grey boxes indicate reported human mutations. (b) Murine T-ALLs harbor one of two classes of deletions within the $5^{\prime}$ region of the Notch 1 gene: (1) $\Delta 1$ - deletion of the proximal promoter including the ATG containing exon 1 allows the transcription of truncated Notch 1 mRNAs driven by a cryptic $3^{\prime}$ promoter located near exon 25 . Normally, Ikaros and/or E2A repress the cryptic $3^{\prime}$ promoter. Loss of Ikaros results in chromatin remodeling and permits facilitated transcription from the $3^{\prime}$ promoter; (2) $\Delta 2$ - deletion of the sequences between exon 1 and exon $26-27$ in which transcription is initiated through the endogenous Notch 1 promoter. Red lines indicate the transcripts produced by the class $\Delta 1$ and $\Delta 2$ deletions. Both classes of mRNA transcripts initiate protein synthesis at a conserved methionine residue (M1727), giving rise to a truncated dominant Notch1 receptor.

lines that were Notch-dependent. These data suggest that the common Notch1 mutations found in human T-ALL are likely to be secondary events that contribute to primary oncogenic hits to accelerate tumor progression. However, the tumors arising are still Notch-dependent, because treatment with pharmacological ( $\gamma$-secretase) inhibitors reduced tumor growth. In addition, the growth dependence of T-ALL expressing weak gain-of-function Notch1 alleles, and the strong selective pressure to acquire Notch1 mutations, implied that even weak Notch signals have additional oncogenic roles in development, progression and or maintenance of the disease. Primary among these oncogenic effects are upregulation of $M y c$ (c-Myc) [18-20], activation of mTOR [21], and increased PI(3)-kinase/Akt signaling [22], all of which have been implicated in the sustained growth of T-ALL cells by Notch1 (Figure 3).

\section{Mechanistic differences in oncogenic Notch1 activation in mice and humans}

Notch receptors comprise a large extracellular domain consisting of EGFR and a negative regulatory region (LNR) that prevents Notch proteolytic cleavage and activation in the absence of ligands (Figure 1). Thus, it is not surprising that during human T-ALL progression, there is a strong selection for ligand-independent NOTCH1 mutant alleles. The most common NOTCH1 mutations in human T-ALL are clustered within the hydrophobic core of the $\mathrm{HD}$, allowing for ligand-independent cleavage. By contrast, the most common mutations in murine T-ALL are truncations of the PEST domain that enhance the stability of the truncated protein, but mutations in the HD domain are rare [16]. The mutations found in the PEST domain of murine T-ALLs usually arise in leukemogenic backgrounds, such as deficiencies of E2A [23], p53 [24], or Ikaros [25], as well as Tal1 misexpression [24] or constitutive K-ras signaling $[17,26]$. Because PEST deletions alone are not oncogenic and most of the murine T-ALLs do not carry mutations in the HD domain, the question how ligand-independent Notch1 activation is initiated in these tumors remained to be clarified. Three recent publications shed new light on this question, and defined deletion-based mechanisms, as well as alternative promoter usage, to support ligand-independent signaling in Notch1 induced T-ALL [27-29]. Historically, the first clues came from studies characterizing radiation-induced and $\mathrm{Atm}^{-1-} \mathrm{T}$ ALLs, which carried deletion breakpoints at the $5^{\prime}$ end of the Notch 1 gene, and the observation that some tumors generated abnormal, short Notch1 transcripts through illegitimate V(D)J recombination [30-32]. Therefore, a panel of murine T-ALL cell lines was examined, revealing that most of them harbor deletions within the $5^{\prime}$ region of the Notch 1 gene. These deletions can be divided into two classes: (a) deletions that remove exon 1 and the proximal promoter, and that are RAG-mediated, and (b) deletions that remove sequences between exon 1 and 26 to 28 , and that are RAG-independent (Figure $2 \mathrm{~b}$ ). The RAG-mediated deletions activate an internal promoter that lies within or adjacent to exon 25 , and drives the expression of $5^{\prime}$ deleted 


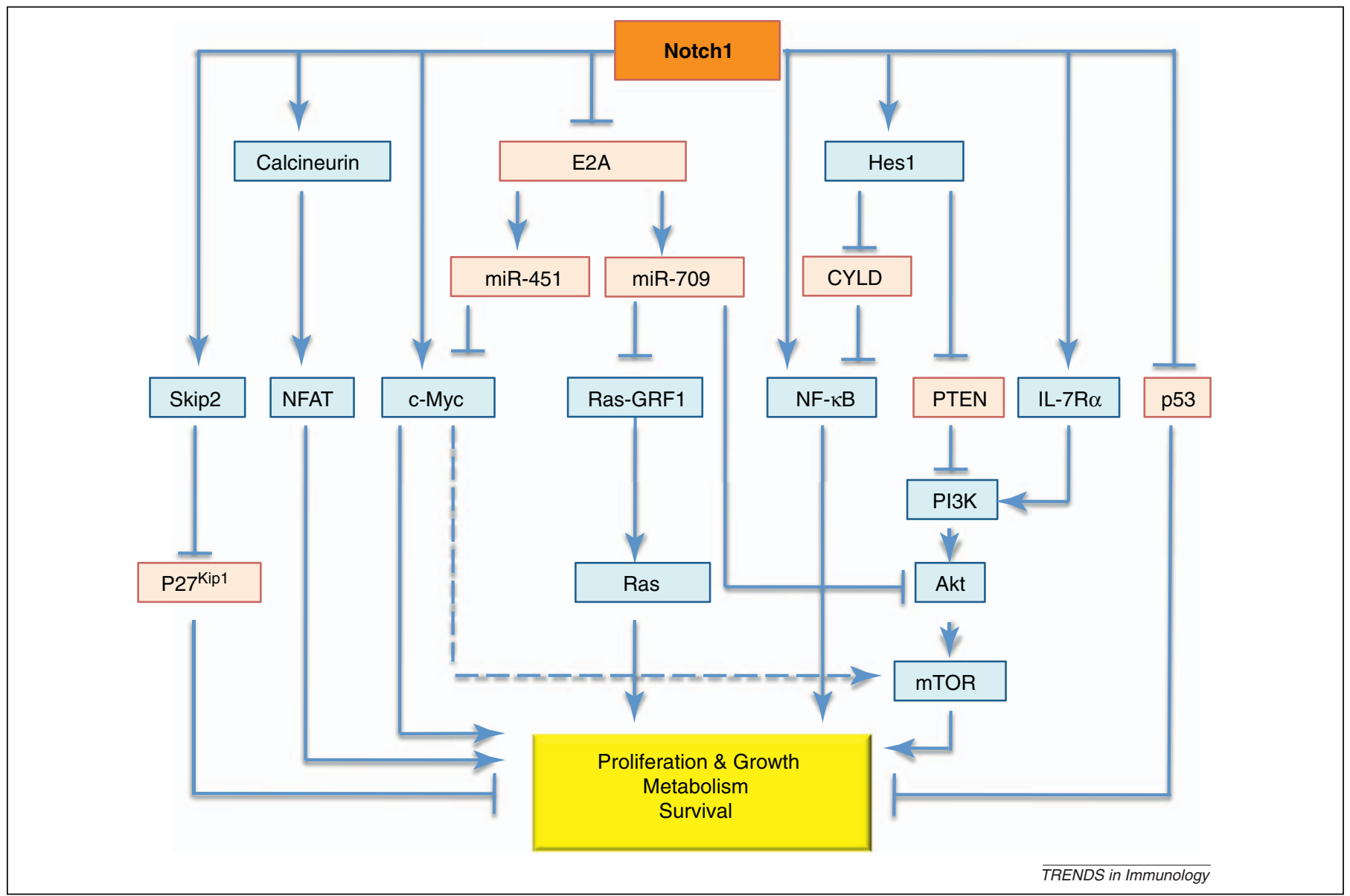

Figure 3. Downstream signaling pathways and molecules implicated in Notch1-mediated T-ALL. The signaling pathways of activated Notch1 in T-ALL are shown. Indicated are positive (arrows) and negative (blocking bars) regulatory interactions contributing to the development and/or maintenance of T-ALL. Growth promoting signaling pathways and molecules are highlighted in blue, whereas growth inhibitory molecules are shown in red. The function of growth inhibitory molecules is often lost in T-ALL,

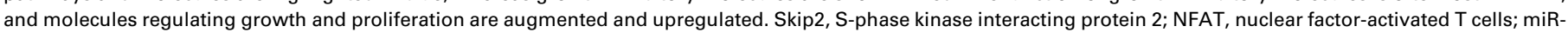
451, micro RNA-451, miR-709, micro RNA-709; Ras-GFR1, Ras protein-specific quanine nucleotide-releasing factor 1; CYLD - Cylindromatosis susceptibility gene; NF-kB,

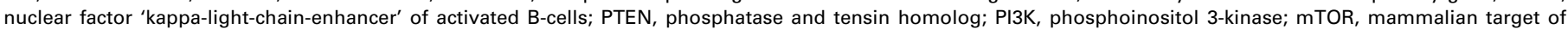
rapamycin; IL-7R $\alpha$, Interleukin-7 receptor alpha.

Notch1 transcripts. ChIP-Seq data-mining analysis revealed that RAG2 associates with the $5^{\prime}$ end of Notch1 in normal thymocytes [28]. Presumably, once RAG2 binds the Notch1 promoter it can recruit RAG1, forming a functional recombinase that mediates illegitimate recombination events because of the presence of ectopic RAG signal sequences. The second type of deletions that remove the region from exon 2 to exon 26-28 occur less frequently, leading to expression of aberrant splice variants from the $5^{\prime}$ proximal promoter. Although the mechanism for the acquisition of these deletions is not clear, they can arise through random DNA breaks and non-homologous endjoining reactions. Nevertheless, the aberrant transcripts of both classes of deletions initiate translation at a conserved methionine residue (M1727) in the N-terminal region of the transmembrane domain of Notch1 just upstream of the $\gamma$-secretase cleavage site (Figure $2 b$ ). This results in the generation of a truncated, dominant, active Notch1 protein that is still transported to the cell surface, and therefore requires $\gamma$-secretase cleavage. This is in line with findings that murine T-ALLs are highly sensitive to $\gamma$-secretase inhibition [28].

Two parallel studies demonstrate that loss of Ikaros also potentiates strongly the transcription of Notch1 alleles harboring $5^{\prime}$ deletions $[27,29]$. Ikaros can bind to the same DNA-binding sequence as RBPJк, and thereby represses Notch target genes [33]. Although inactivating Ikaros mutations are rare in human T-ALL, they occur frequently in mice [34]. Loss-of-function Ikaros mutations cooperate with activating Notch mutations to promote murine TALL, suggesting that Ikaros deficiency leads to enhanced Notch target gene expression $[25,35]$. Supporting this idea are data generated by conditional inactivation of RBPJK that delayed markedly the onset of T-ALL in Ikaros-deficient mice expressing hypomorphic Ikaros. Thus, T-ALL induced by loss of Ikaros requires canonical Notch signaling. Surprisingly, the onset of T-ALL was enhanced markedly when Ikaros-deficient mice were intercrossed with conditional gene targeted mice for Notch1, in which the $5^{\prime}$ end of the Notch 1 gene was deleted by the expression of a T cell-specific Cre recombinase [27,29]. This deletion also activates the same cryptic promoter in exon 25 of Notch1 that was responsible for generating aberrant transcripts in tumors with RAG-mediated deletions [28]. These mice expressed high levels of an abnormal short Notch1 transcript encoding a highly active, truncated Notch1 protein, which frequently accumulated mutations within the PEST domain [27]. Epigenetic studies of the Notch1 locus 
revealed that loss of Ikaros leads to a permissive chromatin configuration and, thus, to the derepression of cryptic promoters present between exon 25 and 27 . This results in the generation of aberrant short Notch1 transcripts, which initiate protein translation at the conserved methionine residue M1727 [27,29]. Therefore, the presence of Ikaros at binding sites within the Notch1 gene locus is required to restrict chromatin accessibility and allows proper gene regulation. Sequence analysis of these cryptic promoter regions within the Notch1 gene revealed the presence of multiple E2A binding sites within or near the Ikaros binding sites. This would suggest that loss of E2A and Ikaros could participate in a common mechanism of transformation generating aberrant Notch1 transcripts in T-ALL that carry deletions of the $5^{\prime}$ region of the Notch 1 gene. Indeed, Notch1 promoter usage in E2A-deficient leukemic cells resulted predominantly in alternative Notch1 transcripts [29]. As a result, these studies indicate that Ikaros, E2A and possibly other proteins restrict chromatin accessibility, thereby suppressing transcriptional initiation from the $3^{\prime}$ end of Notch1, and thus act as tumor suppressors under physiological conditions.

\section{Affected signaling pathways by aberrant Notch signaling}

It has long been known that Notch1 signaling is essential for normal $\mathrm{T}$ cell fate specification, as well as thymocyte maturation and progression through and beyond the $\beta$ selection checkpoint. Thus, it is not surprising that genes and signaling pathways identified downstream of Notch1 in T-ALL cell lines and in vivo mouse models are linked to their physiological role in normal $\mathrm{T}$ cell development. The function of aberrant Notch1 signaling in T-ALL can therefore be inferred from its normal purpose. In the context of $T$ cell transformation, signaling through the Notch1 receptor elicits a program of gene expression that supports cell survival and growth, and represses cell cycle arrest and apoptosis. Novel and controversial insights for some of the key players will be discussed below and are highlighted in Figure 2.

The best-characterized direct target genes include the bHLH transcriptional repressor Hes1 $[18,20,36-38]$ and the transcription factor c-Myc [18-20,39]. Important pathways activated through Notch1 signaling include the PI(3)kinase/Akt [22,40,41] and mTOR [21,42] signaling cascades. Recently, Hes1 was shown to be a key regulator in the induction and maintenance of T-ALL $[37,38,43]$. The use of conditional loss-of-function studies revealed that the transcriptional repressor Hes1 is required for efficient $T$ cell development, and in parallel, Hes 1 is also crucial for the development and maintenance of Notch1-induced murine T-ALL [38]. T-ALL cells seem to require persistent Hes1 expression, since Hes1-deficient cells disappear out of the tumor cell population. T-ALL dependence on Hes1 was not only limited to the mouse model, because lentivirusmediated knockdown of Hes1 in human T-ALL cell lines resulted in a severe block in proliferation followed by increased cell death. These studies indicated that Notch1-induced T-ALL are Hes1 dependent, and that Hes1 might have a conserved function in human and mouse T-ALL [38]. Several suggestions for how Hes1 exerts mechanistically its function in T-ALL have been made; nevertheless this issue seems to be unresolved and needs further investigation. Using fly and human T-ALL cell lines, one study implicated Hes 1 in the transcriptional repression of PTEN expression [22]. PTEN is an important tumor suppressor that counteracts the PI(3)-kinase activity, and thereby negatively regulates the Akt/mTOR pathway (Figure 3). However, in a T-ALL mouse model with conditional Hes1 deficiency, PTEN expression and phospho-Akt levels were unchanged, suggesting that other Notch target genes might be able to compensate for the loss of Hes1 [21]. Thus, the negative effect of Hes1 deficiency on T-ALL development and maintenance must be mediated through other target proteins. Recently, another study implied that Hes1 might repress the deubiquitinase CYLD, which is a negative IKK complex regulator, and thereby sustains NF-кB activation in T-ALL [37,43] (Figure 3). CYLD was shown originally to be a tumor suppressor in the skin, and mutations have been identified in familial cylindromatosis [44]. The fact that cylindromatosis patients do not have an increased risk to develop TALL, and that CYLD mutations have not been identified in T-ALL patients, indicate that Notch signaling, at least in part, must be able to maintain high NF- $\mathrm{KB}$ signaling through alternative pathways.

In normal thymocytes, expression of the proto-oncogene $c$-myc directly correlates with Notch receptor expression, and thus peaks during $\beta$-selection. c-Myc is also a consistently expressed downstream target of NICD in human and mouse T-ALL cell lines (Figure 3). Inhibition of Notch signaling in these cell lines downregulates rapidly c-Myc, and some T-ALL cell lines can be rescued from loss of Notch signaling by enforced overexpression of c-Myc alone [18$21,45]$. However, murine T-ALL cell lines that depend upon ectopic c-Myc overexpression can be rescued from Myc withdrawal through NICD1 expression, which induces sufficient levels of endogenous c-Myc [18]. Moreover, a model for a feed-forward loop, through which Notch and c-Myc reinforce the expression of genes required for growth of leukemic cells, has been established recently [20]. These results suggest that Notch-mediated c-Myc expression is crucial for the maintenance of T-ALL. This view was recently challenged by a study employing a tetracycline inducible mouse model for T-ALL, in which c-Myc expression can be turned off in the presence of constitutive NICD or vice versa. In this experimental setting, continuous expression of NICD, but not c-Myc was required for the maintenance of T-ALL, indicating that c-Myc is incapable of maintaining the murine T-ALL tumors in the absence of NICD [46]. These results contrast most of the data obtained with T-ALL cell lines. It is possible that this controversy might be caused by differences in c-Myc expression levels used in both experimental systems. It is conceivable that T-ALL maintenance requires a certain threshold of c-Myc expression, which might have been reached in one but not the other experimental setting.

Although the complex crosstalk between Notch, the signaling pathways and the molecules discussed above, and the additional interactions outlined in Figure 2 are only understood incompletely, it emphasizes the pleiotropic functions of Notch1 signaling. These functions are, to a 
large extent, reflected as well in its physiological role during $\mathrm{T}$ cell development.

\section{Tumor-promoting and suppressive miRNA in Notch- induced T-ALL}

Micro RNAs (miRNAs), small noncoding RNAs of 19-22 nucleotides, play crucial roles in the translational regulation of protein expression by posttranscriptional silencing. miRNAs are fundamental regulators of physiologically significant cellular processes, and thus, it is not surprising that the deregulation of miRNAs seems to play an important role in human cancers. It has become obvious that miRNAs act as crucial regulators during tumorigenesis, either as oncogenes, as tumor suppressors or even as both, depending on the phase of tumor progression. A role for miRNAs was attributed to affect the normal developmental transitions in T cells through the modulation of TCR signaling strength [44]; however, the involvement of miRNAs in T-ALL has only been shown recently. A novel translocation targeting the miR-17-19 cluster was reported to coincide with a rearrangement, which in turn activates Notch1 [47], thereby identifying the interaction between NOTCH1 and miR-19 as a relevant pathogenic mechanism in T-ALL. Coexistence of two translocations activating the NOTCH1 gene $(\mathrm{t}(9 ; 14)(\mathrm{q} 34 ; \mathrm{q} 11))$ and targeting the $17-19$ cluster $(\mathrm{t}(13 ; 14)(\mathrm{q} 32 ; \mathrm{q} 11))$ was found in the same leukemic clone. Mechanistically, miR-19 acts on several negative regulators of the PI(3)-kinase pathway, which regulate cell survival in lymphocytes and thus contribute to leukemic progression.

By contrast, miRNAs can also act as tumor suppressors, and recent studies associate tumor suppressive functions during tumor maintenance and metastasis. Examples of miRNAs with tumor suppressing activities include miR15a, miR-16-1 and let7 (reviewed in [43]). In a very recent publication, two novel miRNAs, miR-451 and miR-709, were identified in NICD1-overexpressing $\mathrm{CD} 4^{+} \mathrm{CD} 8^{+} \mathrm{T}$ cells through miRNA expression profiling [48]. Both miRNAs are transcriptional targets of the bHLH E2A tumor suppressor, which itself is degraded upon NICD1 induction in murine T-ALL cells $[39,45]$. The $c-M y c$ oncogene is a target of both miRNAs in mouse T-ALL and human T-ALL cell lines, whereas the miR-709, which has no human homologue, also represses efficiently the oncogenes Akt and Ras-GRF1 in murine T-ALL cells. The concomitant expression of both miRNAs - miR-451 and miR-709 - could block efficiently tumor induction in a retroviral NICDinduced T-ALL mouse model. However, once the NICD1 tumor was established in the mouse, reexpression of miR451 and miR-709 at physiological levels could only slow tumor progression slightly, because of a counter-selection against cells that expressed increased amounts of two miRNAs. It was suggested that increased Notch activity facilitates the degradation of E2A that would lead to the transcriptional downregulation of miR-451 and miR-709. This, in turn, would lead to the derepression of $c-m y c$ and $A k t$ and possibly increased Ras signaling (Figure 3), and thus promote T-ALL progression. The expression of miR451 alone in human T-ALL cell lines had no effect on the growth of these cells, and could only inhibit the cell growth in combination with a $\gamma$-secretase inhibitor (MRK-003).
This implies that miR-451 alone is not able to exert enough tumor suppressive activity, and will most probably work in concert with other regulatory mechanisms to control cell growth and proliferation.

Taken together, the two publications discussed above reveal another facet of control mechanisms in human TALL bearing NOTCH1 mutations. However, the mechanistic role of these miRNAs, and possibly others, awaits further clarification.

\section{Concluding remarks}

Although T-ALL is a heterogeneous disease in which multiple genetic aberrations cooperate to deregulate proliferation, differentiation and survival of immature thymocytes, more than half of the patients carry activating mutations within the NOTCH1 gene, highlighting its central role in the disease. The oncogenic role of Notch 1 is a reflection of its physiological function during normal $\mathrm{T}$ cell development. Notch1 is a master regulator for T cell lineage commitment and maturation. However, if Notch1 signaling is not appropriately regulated and shut off at a precise stage during thymocyte development, it exerts oncogenic functions. This will lead to sustained activation of proto-oncogenes and repression of tumor suppressors, in part also by regulating microRNA expression. Recent findings reveal substantial differences in the acquisition of activating Notch1 mutations between murine and human T-ALL. These probably reflect differences in the Notch1 promoter, which favor deletions within the $5^{\prime}$ region of the Notch1 gene in mouse T-ALL. Despite those differences, Notch1 itself, as well some of its downstream molecules, represent prime targets for developing novel anticancer therapies to fight T-ALL.

\section{Acknowledgements}

This work was supported in part by the Swiss National Science Foundation and Oncosuisse.

\section{References}

1 Pui, C.H. and Evans, W.E. (2006) Treatment of acute lymphoblastic leukemia. N. Engl. J. Med. 354, 166-178

2 Van Vlierberghe, P. et al. (2008) Molecular-genetic insights in paediatric T-cell acute lymphoblastic leukaemia. Br. J. Haematol. 143, 153-168

3 Soulier, J. et al. (2005) HOXA genes are included in genetic and biologic networks defining human acute T-cell leukemia (T-ALL). Blood 106, 274-286

4 Ferrando, A.A. et al. (2002) Gene expression signatures define novel oncogenic pathways in $\mathrm{T}$ cell acute lymphoblastic leukemia. Cancer Cell 1, 75-87

5 Bray, S.J. (2006) Notch signalling: a simple pathway becomes complex. Nat. Rev. Mol. Cell Biol. 7, 678-689

6 Ellisen, L.W. et al. (1991) TAN-1, the human homolog of the Drosophila notch gene, is broken by chromosomal translocations in $\mathrm{T}$ lymphoblastic neoplasms. Cell 66, 649-661

7 Reynolds, T.C. et al. (1987) Analysis of DNA surrounding the breakpoints of chromosomal translocations involving the beta T cell receptor gene in human lymphoblastic neoplasms. Cell 50, 107-117

8 Capobianco, A.J. et al. (1997) Neoplastic transformation by truncated alleles of human NOTCH1/TAN1 and NOTCH2. Mol. Cell. Biol. 17, 6265-6273

9 Pear, W.S. et al. (1996) Exclusive development of T cell neoplasms in mice transplanted with bone marrow expressing activated Notch alleles. J. Exp. Med. 183, 2283-2291

$10 \mathrm{Ma}$, S.K. et al. (1999) Cytogenetics and molecular genetics of childhood leukemia. Hematol. Oncol. 17, 91-105 
11 Weng, A.P. et al. (2004) Activating mutations of NOTCH1 in human T cell acute lymphoblastic leukemia. Science 306, 269-271

12 Chiang, M.Y. et al. (2006) Identification of a conserved negative regulatory sequence that influences the leukemogenic activity of NOTCH1. Mol. Cell. Biol. 26, 6261-6271

13 Malecki, M.J. et al. (2006) Leukemia-associated mutations within the NOTCH1 heterodimerization domain fall into at least two distinct mechanistic classes. Mol. Cell. Biol. 26, 4642-4651

14 Bellavia, D. et al. (2000) Constitutive activation of NF-kappaB and Tcell leukemia/lymphoma in Notch3 transgenic mice. EMBO J. 19, 3337-3348

15 Robey, E. et al. (1996) An activated form of Notch influences the choice between CD4 and CD8 T cell lineages. Cell 87, 483-492

16 Aster, J.C. et al. (2008) Notch signaling in leukemia. Annu. Rev. Pathol. $3,587-613$

17 Chiang, M.Y. et al. (2008) Leukemia-associated NOTCH1 alleles are weak tumor initiators but accelerate K-ras-initiated leukemia. J. Clin. Invest. 118, 3181-3194

18 Weng, A.P.et al. (2006) c-Myc is an important direct target of Notch1 in Tcell acute lymphoblastic leukemia/lymphoma. Genes Dev. 20, 2096-2109

19 Sharma, V.M. et al. (2006) Notch1 contributes to mouse T-cell leukemia by directly inducing the expression of c-myc. Mol. Cell. Biol. 26, 8022-8031

20 Palomero, T. et al. (2006) NOTCH1 directly regulates c-MYC and activates a feed-forward-loop transcriptional network promoting leukemic cell growth. PNAS 103, 18261-18266

21 Chan, S.M. et al. (2007) Notch signals positively regulate activity of the mTOR pathway in T-cell acute lymphoblastic leukemia. Blood 110, 278-286

22 Palomero, T. et al. (2007) Mutational loss of PTEN induces resistance to NOTCH1 inhibition in T-cell leukemia. Nat. Med. 13, 1203-1210

23 Reschly, E.J. et al. (2006) Notch1 promotes survival of E2A-deficient T cell lymphomas through pre-T cell receptor-dependent and independent mechanisms. Blood 107, 4115-4121

24 O'Neil, J. et al. (2006) Activating Notch1 mutations in mouse models of T-ALL. Blood 107, 781-785

25 Dumortier, A. et al. (2006) Notch activation is an early and critical event during T-cell leukemogenesis in Ikaros-deficient mice. Mol. Cell. Biol. 26, 209-220

26 Kindler, T. et al. (2008) K-RasG12D-induced T-cell lymphoblastic lymphoma/leukemias harbor Notch1 mutations and are sensitive to gamma-secretase inhibitors. Blood 112, 3373-3382

27 Jeannet, R. et al. (2010) Oncogenic activation of the Notch1 gene by deletion of its promoter in Ikaros-deficient T-ALL. Blood 116, 5443-5454

28 Ashworth, T.D. et al. (2010) Deletion-based mechanisms of Notch1 activation in T-ALL: key roles for RAG recombinase and a conserved internal translational start site in Notch1. Blood 116, 5455-5464

29 Gomez-del Arco, P. et al. (2010) Alternative promoter usage at the Notch1 locus supports ligand-independent signaling in $\mathrm{T}$ cell development and leukemogenesis. Immunity 33, 685-698

30 Tsuji, H. et al. (2004) Involvement of illegitimate V(D)J recombination or microhomology-mediated nonhomologous end-joining in the formation of intragenic deletions of the Notch1 gene in mouse thymic lymphomas. Cancer Res. $64,8882-8890$

31 Tsuji, H. et al. (2009) Rag-dependent and Rag-independent mechanisms of Notch1 rearrangement in thymic lymphomas of $\operatorname{Atm}(-/-)$ and scid mice. Mutat. Res. 660, 22-32

32 Tsuji, H. et al. (2003) Radiation-induced deletions in the $5^{\prime}$ end region of Notch1 lead to the formation of truncated proteins and are involved in the development of mouse thymic lymphomas. Carcinogenesis 24, $1257-1268$

33 Chari, S. and Winandy, S. (2008) Ikaros regulates Notch target gene expression in developing thymocytes. J. Immunol. 181, 6265-6274

34 Marcais, A. et al. (2010) Genetic inactivation of Ikaros is a rare event in human T-ALL. Leuk. Res. 34, 426-429

35 Beverly, L.J. and Capobianco, A.J. (2003) Perturbation of Ikaros isoform selection by MLV integration is a cooperative event in Notch(IC)-induced T cell leukemogenesis. Cancer Cell 3, 551-564

36 Dudley, D.D. et al. (2009) Hes1 potentiates T cell lymphomagenesis by up-regulating a subset of notch target genes. PLOS ONE 4, e6678

37 Espinosa, L. et al. (2010) The Notch/Hes1 pathway sustains NFkappaB activation through CYLD repression in $\mathrm{T}$ cell leukemia. Cancer Cell 18, 268-281
38 Wendorff, A.A. et al. (2010) Hes1 is a critical but context-dependent mediator of canonical Notch signaling in lymphocyte development and transformation. Immunity 33, 671-684

$39 \mathrm{Li}, \mathrm{X}$. et al. (2008) Oncogenesis of T-ALL and nonmalignant consequences of overexpressing intracellular NOTCH1. J. Exp. Med. 205, 2851-2861

40 Ciofani, M. and Zuniga-Pflucker, J.C. (2005) Notch promotes survival of pre-T cells at the beta-selection checkpoint by regulating cellular metabolism. Nat. Immunol. 6, 881-888

41 Sade, H. et al. (2004) The anti-apoptotic effect of Notch-1 requires p56lck-dependent, Akt/PKB-mediated signaling in T cells. J. Biol. Chem. 279, 2937-2944

42 Cullion, K. et al. (2009) Targeting the Notch1 and mTOR pathways in a mouse T-ALL model. Blood 113, 6172-6181

43 D'Altri, T. et al. (2011) Hes1 expression and CYLD repression are essential events downstream of Notch1 in T-cell leukemia. Cell Cycle 10, 1031-1036

44 Bignell, G.R. et al. (2000) Identification of the familial cylindromatosis tumour-suppressor gene. Nat. Genet. 25, 160-165

45 Sharma, V.M. et al. (2007) The Notch1/c-Myc pathway in T cell leukemia. Cell Cycle 6, 927-930

46 Demarest, R.M. et al. (2011) Notch is oncogenic dominant in T-cell acute lymphoblastic leukemia. Blood 117, 2901-2909

47 Mavrakis, K.J. et al. (2010) Genome-wide RNA-mediated interference screen identifies miR-19 targets in Notch-induced T-cell acute lymphoblastic leukaemia. Nat. Cell Biol. 12, 372-379

$48 \mathrm{Li}$, X.et al. (2011) Repression of tumor suppressor miR-451 is essential for NOTCH1-induced oncogenesis in T-ALL. J. Exp. Med. 208, 663-675

49 Bash, R.O. et al. (1995) Does activation of the TAL1 gene occur in a majority of patients with T-cell acute lymphoblastic leukemia? A pediatric oncology group study. Blood 86, 666-676

50 van Grotel, M. et al. (2006) The outcome of molecular-cytogenetic subgroups in pediatric T-cell acute lymphoblastic leukemia: a retrospective study of patients treated according to DCOG or COALL protocols. Haematologica 91, 1212-1221

51 Hwang, L.Y. and Baer, R.J. (1995) The role of chromosome translocations in T cell acute leukemia. Curr. Opin. Immunol. 7, 659-664

52 Wang, J. et al. (2000) The $\mathrm{t}(14 ; 21)(\mathrm{q} 11.2 ; \mathrm{q} 22)$ chromosomal translocation associated with T-cell acute lymphoblastic leukemia activates the BHLHB1 gene. Proc. Natl. Acad. Sci. U.S.A. 97, 3497-3502

53 Erikson, J. et al. (1986) Deregulation of c-myc by translocation of the alpha-locus of the T-cell receptor in T-cell leukemias. Science 232, 884-886

54 McKeithan, T.W. et al. (1986) Molecular cloning of the breakpoint junction of a human chromosomal 8;14 translocation involving the T-cell receptor alpha-chain gene and sequences on the $3^{\prime}$ side of MYC. Proc. Natl. Acad. Sci. U.S.A. 83, 6636-6640

55 Shima, E.A. et al. (1986) Gene encoding the alpha chain of the T-cell receptor is moved immediately downstream of c-myc in a chromosomal 8;14 translocation in a cell line from a human T-cell leukemia. Proc. Natl. Acad. Sci. U.S.A. 83, 3439-3443

56 Boehm, T. et al. (1991) The rhombotin family of cysteine-rich LIMdomain oncogenes: distinct members are involved in T-cell translocations to human chromosomes 11p15 and 11p13. Proc. Natl. Acad. Sci. U.S.A. 88, 4367-4371

57 Royer-Pokora, B. et al. (1991) TTG-2, a new gene encoding a cysteinerich protein with the LIM motif, is overexpressed in acute T-cell leukaemia with the $\mathrm{t}(11 ; 14)(\mathrm{p} 13 ; \mathrm{q} 11)$. Oncogene $6,1887-1893$

58 Royer-Pokora, B. et al. (1995) The TTG-2/RBTN2 T cell oncogene encodes two alternative transcripts from two promoters: the distal promoter is removed by most $11 \mathrm{p} 13$ translocations in acute $\mathrm{T}$ cell leukaemias (T-ALL). Oncogene 10, 1353-1360

59 Sanchez-Garcia, I. and Rabbitts, T.H. (1993) LIM domain proteins in leukaemia and development. Semin. Cancer Biol. 4, 349-358

60 Cave, H. et al. (2004) Clinical significance of HOX11L2 expression linked to $\mathrm{t}(5 ; 14)(\mathrm{q} 35 ; \mathrm{q} 32)$, of HOX11 expression, and of SIL-TAL fusion in childhood T-cell malignancies: results of EORTC studies 58881 and 58951. Blood 103, 442-450

61 Hatano, M. et al. (1991) Deregulation of a homeobox gene, HOX11, by the $\mathrm{t}(10 ; 14)$ in T cell leukemia. Science $253,79-82$ 
$62 \mathrm{Lu}, \mathrm{M}$. et al. (1991) The tcl-3 proto-oncogene altered by chromosomal translocation in T-cell leukemia codes for a homeobox protein. EMBO J. 10, 2905-2910

63 Ballerini, P. et al. (2002) HOX11L2 expression defines a clinical subtype of pediatric T-ALL associated with poor prognosis. Blood 100, 991-997

64 Clappier, E. et al. (2007) The C-MYB locus is involved in chromosomal translocation and genomic duplications in human T-cell acute leukemia (T-ALL), the translocation defining a new T-ALL subtype in very young children. Blood 110, 1251-1261

65 Batova, A. et al. (1997) Frequent and selective methylation of p15 and deletion of both p15 and p16 in T-cell acute lymphoblastic leukemia. Cancer Res. 57, 832-836

66 Diccianni, M.B. et al. (1997) Shortened survival after relapse in T-cell acute lymphoblastic leukemia patients with p16/p15 deletions. Leuk. Res. 21, 549-558

67 Cauwelier, B. et al. (2007) Clinical, cytogenetic and molecular characteristics of 14 T-ALL patients carrying the TCRbeta-HOXA rearrangement: a study of the Groupe Francophone de Cytogenetique Hematologique. Leukemia 21, 121-128

68 Lahortiga, I. et al. (2007) Duplication of the MYB oncogene in T cell acute lymphoblastic leukemia. Nat. Genet. 39, 593-595

69 De Keersmaecker, K. et al. (2005) Fusion of EML1 to ABL1 in T-cell acute lymphoblastic leukemia with cryptic t(9;14)(q34;q32). Blood 105, 4849-4852

70 Van Limbergen, H. et al. (2001) Molecular cytogenetic and clinical findings in ETV6/ABL1-positive leukemia. Genes Chromosomes Cancer 30, 274-282

71 Burmeister, T. et al. (2006) NUP214-ABL1 in adult T-ALL: the GMALL study group experience. Blood 108, 3556-3559

72 Graux, C. et al. (2004) Fusion of NUP214 to ABL1 on amplified episomes in T-cell acute lymphoblastic leukemia. Nat. Genet. 36, 1084-1089

73 Ferrando, A.A. et al. (2003) Gene expression signatures in MLLrearranged T-lineage and B-precursor acute leukemias: dominance of HOX dysregulation. Blood 102, 262-268
74 O'Neil, J. et al. (2007) FBW7 mutations in leukemic cells mediate NOTCH pathway activation and resistance to gamma-secretase inhibitors. J. Exp. Med. 204, 1813-1824

75 Thompson, B.J.et al. (2007) The SCFFBW7 ubiquitin ligase complex as a tumor suppressor in T cell leukemia. J. Exp. Med. 204, 1825-1835

76 Gutierrez, A. et al. (2009) High frequency of PTEN, PI3K, and AKT abnormalities in T-cell acute lymphoblastic leukemia. Blood 114 $647-650$

77 Medyouf, H. et al. (2009) Acute T-cell leukemias remain dependent on Notch signaling despite PTEN and INK4A/ARF loss. Blood

78 Seiffert, D. et al. (2000) Presenilin-1 and -2 are molecular targets for gamma-secretase inhibitors. J. Biol. Chem. 275, 34086-34091

79 Milano, J. et al. (2004) Modulation of notch processing by gammasecretase inhibitors causes intestinal goblet cell metaplasia and induction of genes known to specify gut secretory lineage differentiation. Toxicol. Sci. 82, 341-358

80 Riccio, O. et al. (2008) Loss of intestinal crypt progenitor cells owing to inactivation of both Notch1 and Notch2 is accompanied by derepression of CDK inhibitors p27(Kip1) and p57(Kip2). EMBO Rep. 9, 377-383

81 Real, P.J. et al. (2009) Gamma-secretase inhibitors reverse glucocorticoid resistance in $\mathrm{T}$ cell acute lymphoblastic leukemia. Nat. Med. 15, 50-58

$82 \mathrm{Li}, \mathrm{K}$. et al. (2008) Modulation of Notch signaling by antibodies specific for the extracellular negative regulatory region of NOTCH3. J. Biol. Chem. 283, 8046-8054

$83 \mathrm{Wu}$, Y. et al. (2010) Therapeutic antibody targeting of individual Notch receptors. Nature 464, 1052-1057

84 Noguera-Troise, I. et al. (2006) Blockade of Dll4 inhibits tumour growth by promoting non-productive angiogenesis. Nature 444, 1032-1037

85 Ridgway, J. et al. (2006) Inhibition of Dll4 signalling inhibits tumour growth by deregulating angiogenesis. Nature 444, 1083-1087

86 Aste-Amezaga, M. et al. (2010) Characterization of Notch1 antibodies that inhibit signaling of both normal and mutated Notch1 receptors. PLoS ONE 5, e9094

87 Moellering, R.E. et al. (2009) Direct inhibition of the NOTCH transcription factor complex. Nature 462, 182-188 